\title{
Calidad de vida en adultos con cicatrices queloides
}

\author{
Quality of life in adults with keloid scars
}

\author{
Martha A. Morales-Sánchez*, Christian N. Flores-Ruvalcaba, María L. Peralta-Pedrero, \\ Andrea De Villafranca-Dugelby y Fermín Jurado-Santa Cruz \\ Unidad de Investigación, Centro Dermatológico Dr. Ladislao de la Pascua, Servicios de Salud Pública, Ciudad de México, México
}

\begin{abstract}
Resumen
Introducción: Las cicatrices queloides afectan hasta el 16\% de la población hispana y pueden causar un deterioro en la calidad de vida. La mayoría de los estudios previos han estudiado pacientes con cicatrices hipertróficas secundarias a quemaduras. Objetivo: Determinar la calidad de vida en adultos con cicatrices queloides que acuden a un centro dermatológico. Método: Se realizó un estudio transversal analítico en el Centro Dermatológico Dr. Ladislao de la Pascua, en adultos con diagnóstico de cicatrices queloides. Se midió la calidad de vida mediante el instrumento DLQI (Dermatology Life Quality Index) y se estudiaron las siguientes variables clínicas: edad, sexo, tiempo de evolución, tamaño de la cicatriz, número de cicatrices y topografía afectada. Se aplicó el cuestionario de Beck para el tamizaje de la depresión. Resultados: Se reclutaron 106 adultos con cicatrices queloides (61\% hombres y 39\% mujeres), con una mediana de edad de 26 años (rango intercuartilar: \pm 18 años). El puntaje promedio del DLQI fue de 5.58 (desviación estándar [DE]: \pm 5.528 ), con un máximo obtenido de 22 puntos; dicho puntaje se interpreta como un deterioro mínimo de la calidad de vida. La dimensión que más contribuyó al total del puntaje fue la de síntomas y sentimientos, con el 50.36\%. El número de cicatrices y la edad se asociaron directamente con el puntaje del DLQI. El puntaje promedio obtenido con el cuestionario de Beck fue de 6.45 (DE: \pm 6.46 puntos) y se correlacionó con el puntaje del DLQI. Conclusiones: Las cicatrices queloides tienen un efecto negativo mínimo en la calidad de vida. Dicho efecto se asoció al autorreporte de síntomas depresivos; sin embargo, los resultados de este estudio no permiten atribuirle una relación casual. Se recomienda aplicar el cuestionario de calidad de vida en adultos jóvenes con cicatrices.
\end{abstract}

PALABRAS CLAVE: Cicatriz queloide. Cicatriz. Calidad de vida. Adultos.

\begin{abstract}
Introduction: Keloid scars affect up to $16 \%$ of the Hispanic population and may cause deterioration in the quality of life. Most of the previous studies have focused on patients with hypertrophic scars secondary to burns. Objective: To determine the quality of life in adults with keloid scars that go to a dermatological center. Methods: An analytical cross-sectional study was carried out at Centro Dermatológico Dr. Ladislao de la Pascua in adults with a diagnosis of keloid scars. The quality of life was measured using the Dermatology Life Quality Index (DLQI) and the following clinical variables were studied: age, sex, evolution time, scar size, number of scars and affected topography. The Beck questionnaire for depression was also applied. Results: 106 adults with keloid scars were recruited, 61\% men and 39\% women, with a median age of 26 years (interquartile range: \pm 18 years). The average score of the DLQI was 5.58 (standard deviation [SD]: \pm 5.528 ), with a maximum obtained of 22 points, this score is interpreted as a minimum or small deterioration of the quality of life. The dimension that contributed most to the total score was symptoms and feelings, with $50.36 \%$. The number of scars and age were directly associated with the DLQI score. The average score obtained with the Beck questionnaire was: 6.45 (SD: \pm 6.46 points) and it correlates with the DLQI score. Conclusions: Keloid scars have a minimal negative effect on the quality of life of young adults. This effect
\end{abstract}

\author{
Correspondencia: \\ *Martha A. Morales-Sánchez \\ José María Vértiz, 464 \\ Col. Buenos Aires, Del. Cuauhtémoc \\ C.P. 06780, Ciudad de México, México \\ E-mail: marthamoralessanchez@gmail.com
}

Fecha de recepción: 16-02-2018

Fecha de aceptación: 28-04-2018

DOI://dx.doi.org/10.24875/CIRU.M18000049
Cir Cir. 2018;86:321-326

Contents available at PubMed www.cirugiaycirujanos.com 
was associated with the self-report of depressive symptoms; however, the results of this study do not allow to attribute a causal relationship. It is recommended to apply the quality of life questionnaire in young adults with scars.

KEY WORDS: Keloid scar. Scar. Cicatrix. Quality of life. Adults.

\section{Introducción}

La cicatrización queloide es una alteración en la reparación de las heridas caracterizada por la síntesis exagerada de tejido conectivo en respuesta a traumatismos, quemaduras, procesos inflamatorios e incisiones quirúrgicas. Su incidencia oscila del 4.5 al $16 \%$, predomina en personas de raza negra e hispanos, y se presenta entre los 10 y 30 años de edad'. Se asocia con los HLA B14, B21, BW16, BW35, DR5 y DQW3, y al tipo de sangre $A R^{2}+^{2}$. Se ha reportado una herencia autosómica dominante y autosómica recesiva en casos familiares ${ }^{2}$.

Las cicatrices queloides se desarrollan principalmente en las orejas, el área de la barba, el tronco y los hombros. Su diagnóstico es clínico y deben diferenciarse de la cicatriz hipertrófica, ya que esta permanece confinada a los límites de la herida, mientras que la cicatriz queloide los rebasa e invade la dermis normal ${ }^{3}$. Por su evolución, la cicatrización hipertrófica aparece dentro de las primeras 4 semanas después de la herida y experimenta una regresión espontánea, en contraste con la cicatriz queloide, que se presenta tiempo después y prolifera indefinidamente ${ }^{3}$.

La piel es importante en términos de imagen corporal, y cualquier enfermedad que la afecte puede ocasionar un deterioro en la calidad de vida de los pacientes. La medición de la calidad de vida en pacientes con patología cutánea puede hacerse mediante instrumentos genéricos que proporcionan una idea global de la calidad de vida, o mediante instrumentos específicos como el Índice de Calidad de Vida en Dermatología (DLQI, Dermatology Life Quality Index)4.

Recientemente, un estudio realizado en pacientes con cicatrices queloides encontró que el dolor y el prurito se asocian a una disminución en la calidad de vida, principalmente del bienestar emocional y mental. Dicha disminución en la calidad de vida se correlacionaba con la edad, la visibilidad de la cicatriz, el número de cicatrices, la falta de elasticidad y la irregularidad de la superficie de las cicatrices ${ }^{5}$. También se ha documentado que la calidad de vida mejora en cuanto disminuye el tamaño de la cicatriz debido al tratamiento ${ }^{6}$.

Hasta el momento no existen trabajos en nuestra población sobre la calidad de vida de adultos con cicatrices queloides, por lo que el objetivo principal de este estudio fue determinar la calidad de vida en pacientes que desarrollaron cicatrices queloides y que acudieron a un centro de referencia para enfermedades de la piel para su tratamiento. Como objetivo secundario se determinó la prevalencia de depresión mediante un instrumento de tamizaje autoaplicado, y la correlación de esta con la calidad de vida.

\section{Método}

Se realizó un estudio transversal en adultos con diagnóstico de cicatrices queloides que acudieron al Centro Dermatológico Dr. Ladislao de la Pascua en el periodo de marzo de 2016 a enero 2017. El protocolo fue aprobado por el Comité de Ética en Investigación de la institución y todos los pacientes firmaron un consentimiento informado en caso de que aceptaran participar. Los criterios de inclusión fueron tener una edad mayor de 18 años, diagnóstico clínico de cicatriz queloide y que el motivo de consulta fuera el tratamiento de las cicatrices. Se excluyeron los pacientes con alguna discapacidad visual o motora que les impidiera contestar los cuestionarios del estudio y aquellos que fueran analfabetos.

Un dermatólogo verificaba el diagnóstico clínico de cicatriz queloide y documentaba las siguientes variables sobre las cicatrices: tamaño, topografía, tiempo de evolución, tratamientos previos y atención médica recibida. Posterior a la realización de la historia clínica, los pacientes contestaban de forma independiente el DLQI y el inventario de depresión de Beck. EI DLQI es una escala unidimensional que consta de un cuestionario de 10 preguntas, las cuales incluyen las siguientes dimensiones: 1) síntomas y sentimientos; 2) actividades diarias; 3) ocio; 4) trabajo y vida escolar; 5) relaciones interpersonales, incluyendo la sexualidad; y 6) efectos secundarios del tratamiento ${ }^{4}$. El DLQI se calcula sumando los puntajes de cada pregunta, resultando en un mínimo de 0 y un máximo de $30^{4}$; los puntajes altos se correlacionan con un deterioro de la calidad de vida. El inventario de depresión de Beck ${ }^{7}$ consta de 21 preguntas de opción múltiple con un puntaje mínimo de 0 y máximo de 63 . A mayor puntaje, mayor probabilidad de presentar depresión; 
un puntaje de 1 a 10 se considera normal. Dicho inventario es un instrumento que incluye los criterios mayores para diagnóstico de depresión y es de utilidad para identificar pacientes que requieren atención psiquiátrica.

El tamaño de muestra se calculó de acuerdo con el promedio del DLQI reportado por Reinholz, et al. ${ }^{8}$ en adultos con cicatrices queloides, de 6.06 (desviación estándar [DE]: 4.0) puntos, y se utilizó la fórmula para calcular una media poblacional con valor alfa de 0.01 y una precisión de 1 punto. Los datos se analizaron con el programa SPSS v.19. Las variables cualitativas se reportaron en proporciones, y las cuantitativas con distribución normal mediante promedio y desviación estándar; en caso contrario, se reportaron la mediana y el rango intercuartilar. Las pruebas estadísticas utilizadas fueron las de chi al cuadrado, $t$ de Student y correlación de Pearson, y se consideró significancia estadística un valor de $p<0.05$.

\section{Resultados}

Se reclutaron 106 adultos con diagnóstico de cicatrices queloides, el $61 \%(n=65)$ hombres y el $39 \%$ ( $n=41$ ) mujeres, con una mediana de edad de 26 años (rango intercuartilar: \pm 18 años). El tiempo promedio transcurrido desde el inicio de la enfermedad hasta el diagnóstico fue de 2 años (rango intercuartilar: \pm 4 años). Al momento de acudir a consulta por las cicatrices, el $80.2 \%$ de los pacientes fueron vistos previamente por algún médico, de los cuales el $52.94 \%$ eran dermatólogos. El $55.7 \%(n=59)$ de los pacientes recibió algún tratamiento previo, y el más usado fue el esteroide intralesional $(24.5 \%$ de los pacientes), seguido del gel de silicón (14.2\%).

La etiología de las cicatrices fue: $41.5 \%$ acné, $17.9 \%$ cirugía, $17 \%$ traumatismo, $7.5 \%$ herida, $6.6 \%$ varicela, $5.7 \%$ perforaciones, $1.9 \%$ foliculitis y $1.9 \%$ inmunizaciones. La topografía más frecuente fue el tronco, en el $60.4 \%$ de los casos, seguida de las extremidades superiores con el $16 \%$; el resto de las localizaciones se detallan en la tabla 1.

El $48.1 \%(n=51)$ de las cicatrices se encontraban en áreas visibles. El $32.1 \%$ de los pacientes presentaban solo una cicatriz y el $67.9 \%$ restante múltiples, siendo la mediana 3 cicatrices (rango intercuartilar: \pm 6), con un máximo de 40 por paciente. Respecto al tamaño de las cicatrices, el promedio de largo fue de $3.5 \mathrm{~cm}$ (DE: $2.54 \mathrm{~cm})$, de ancho $1.55 \mathrm{~cm}$ (DE: $1.27 \mathrm{~cm})$ y de altura $2.75 \mathrm{~mm}$ (DE: $1.73 \mathrm{~mm}$ ).
Tabla 1. Topografía de las cicatrices $(n=106)$

\begin{tabular}{lcc}
\hline Topografía & Frecuencia & $\%$ \\
\hline Tronco & 64 & 60.4 \\
Extremidad superior & 17 & 16.0 \\
Oreja & 11 & 10.4 \\
Piel cabelluda & 6 & 5.7 \\
Región de la barba & 3 & 2.8 \\
Barba y tronco & 2 & 1.9 \\
Cuello & 1 & 0.9 \\
Extremidad inferior & 1 & 0.9 \\
Cuello y tronco & 1 & 0.9 \\
\hline
\end{tabular}

Tabla 2. Calidad de vida por dimensión del DLQI

\begin{tabular}{lccc}
\hline Dimensión & Ítems & $\begin{array}{c}\text { Puntaje } \\
\text { Media (DE) }\end{array}$ & $\begin{array}{c}\text { Porcentaje } \\
\text { del total }\end{array}$ \\
\hline Síntomas y sentimientos & $1-2$ & $1.98(1.48)$ & 50.36 \\
Actividades diarias & $3-4$ & $1.27(1.55)$ & 17.94 \\
Ocio & $5-6$ & $1.01(1.47)$ & 12.95 \\
Trabajo y vida escolar & 7 & $0.42(0.63)$ & 08.40 \\
Relaciones interpersonales & $8-9$ & $0.63(1.32)$ & 07.59 \\
$\begin{array}{l}\text { Efectos secundarios de } \\
\text { tratamiento }\end{array}$ & 10 & $0.25(0.74)$ & 02.86 \\
\hline
\end{tabular}

\section{Análisis de la calidad de vida}

El puntaje promedio del DLQl fue de 5.58 (DE: 5.528), con un máximo de 22 puntos; dicho puntaje se interpreta como un deterioro mínimo o pequeño de la calidad de vida. La dimensión que más contribuyó al total del puntaje fue la de síntomas y sentimientos, con el $50.36 \%$, seguida de la de actividades diarias con el $17.94 \%$ y la de ocio con el $12.95 \%$. El puntaje promedio obtenido en cada una de las dimensiones, los ítems evaluados y la contribución al total se muestran en la tabla 2. La distribución de los 106 pacientes de acuerdo con la calificación del DLQI, o grado de afectación de la calidad de vida, se presenta en la figura 1.

Al analizar el puntaje del DLQI según la edad, no se encontró correlación entre ambas variables $(r=-0.12 ; p=0.23)$. La situación fue similar con el tiempo de evolución $(r=-0.125 ; p=0.20)$. Al comparar los puntajes del DLQI de acuerdo con el sexo del paciente no se encontró una diferencia estadísticamente significativa $(t=1.201 ; p=0.233)$. Tampoco se 


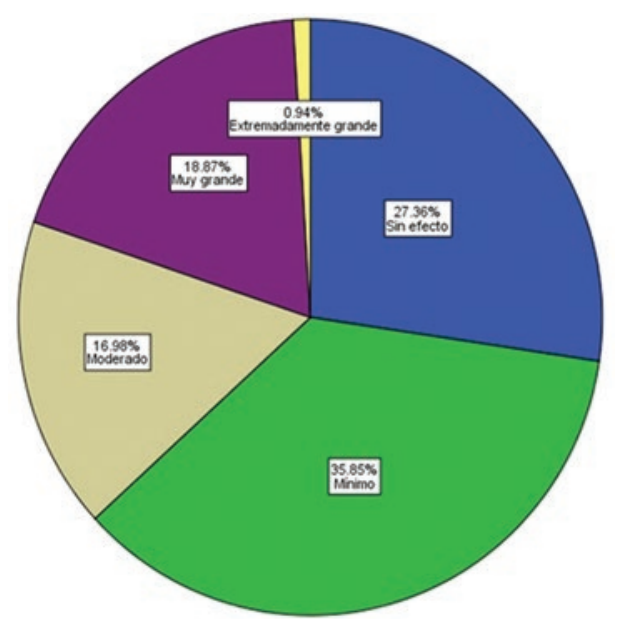

Figura 1. Distribución de los pacientes por grado de afectación de la calidad de vida.

encontró correlación entre el puntaje del DLQI y el tamaño de la cicatriz $(r=-0.123 ; p=0.209)$ o el número de estas $(r=0.172 ; p=0.079)$, ni se evidenciaron diferencias en cuanto a la visibilidad $(r=0.09$; $p=0.361$ ), es decir, si se encontraban en la cabeza o el cuello en comparación con otras topografías.

Al comparar las características de los pacientes que obtuvieron un puntaje alto en el DLQI (> 11 puntos, que significa un gran impacto en la calidad de vida) con las de aquellos que tuvieron puntajes de 0 y 1 (sin impacto en la calidad de vida) se encontró que el número de cicatrices y la edad se asociaban directamente con el puntaje. En el resto de las variables analizadas no se observó una diferencia estadísticamente significativa, tal como se muestra en la tabla 3.

\section{Análisis de la calidad de vida y de la depresión}

El puntaje promedio obtenido con el cuestionario de Beck fue de 6.45 (DE: 6.46 puntos). La distribución de pacientes de acuerdo con el resultado del cuestionario de Beck, en porcentajes, se puede observar en la figura 2. Al comparar los puntajes del DLQI según el grado de depresión de los pacientes se observó que, a mayor puntaje de DLQI, mayores síntomas de depresión reportados por el paciente $(F=5.88$; $p=0.0001$ ), como se muestra en la tabla 4 .

\section{Discusión}

En nuestro estudio, los pacientes obtuvieron un puntaje promedio de calidad de vida de 5.58 (DE: 5.528),
Tabla 3. Variables asociadas a la calidad de vida

\begin{tabular}{lccc}
\hline Variable & $\begin{array}{c}\text { Puntaje DLQI } \\
\mathbf{0 - 1} \\
\text { Media (DE) }\end{array}$ & $\begin{array}{c}\text { Puntaje } \\
\text { DLQI }>\mathbf{1 1} \\
\text { Media (DE) }\end{array}$ & p \\
\hline Numero de cicatrices & $3.24(3.76)$ & $9.9(11.73)$ & 0.020 \\
Edad & $31.24(13.36)$ & $25.24(7.48)$ & 0.049 \\
Volumen $\left(\mathrm{mm}^{3}\right)$ & $206.3(330.1)$ & $148.01(217.81)$ & 0.484 \\
Tiempo de evolución & $3.65(6.07)$ & $2.61(5.47)$ & 0.538 \\
Visibilidad & $48.3 \%$ & $57.14 \%$ & 0.536 \\
Sexo femenino & $34.48 \%$ & $52.38 \%$ & 0.206 \\
\hline
\end{tabular}

Tabla 4. Grado de depresión según el cuestionario de Beck y calidad de vida

\begin{tabular}{lccc}
\hline Nivel de depresión & Rangos & $\begin{array}{c}\text { Puntaje DLQI } \\
\text { Media (DE) }\end{array}$ & p \\
\hline Sin altibajos & 0 & $3.16(3.06)$ & $*$ \\
Altibajos normales & $1-10$ & $4.83(4.71)$ & 0.075 \\
Leve perturbación del & $11-16$ & $9.14(6.66)$ & 0.006 \\
estado de ánimo & & & \\
Depresión intermitente & $17-20$ & $8.83(3.86)$ & 0.001 \\
Depresión moderada & $21-30$ & $13.67(11.93)$ & 0.002 \\
Depresión grave & $31-40$ & 0 & - \\
Depresión extrema & $>40$ & 0 & - \\
\hline
\end{tabular}

valor similar al reportado por Chuan y Goh $\left.{ }^{9} 5.62 \pm 4.3\right)$. Según los resultados, se observa que el deterioro de la calidad de vida de nuestros pacientes con cicatrices queloides es mínimo, al igual que en otras patologías como psoriasis, vitíligo, rosácea, acné y dermatitis atópica. El dominio de la calidad de vida que resultó más afectado fue el de síntomas y sentimientos, que correspondió al $50.36 \%$ del total del puntaje del cuestionario DLQI, lo cual concuerda con lo publicado previamente por Reinholz, et al. ${ }^{8}$ en este tipo de pacientes. Este deterioro de la calidad de vida es atribuible al impacto que tienen las cicatrices queloides en la esfera psicológica del paciente, lo cual, de acuerdo con nuestros resultados, no se asocia a un deterioro importante en los dominios de actividades diarias, ocio, trabajo o vida escolar, relaciones interpersonales y efectos secundarios de los tratamientos.

Al contrastar las características de nuestra muestra y clasificarla en dos grupos, sin deterioro y con deterioro importante de la calidad de vida (0-1 puntos $y>11$ puntos, respectivamente), encontramos que los pacientes con deterioro de la calidad de vida tenían 


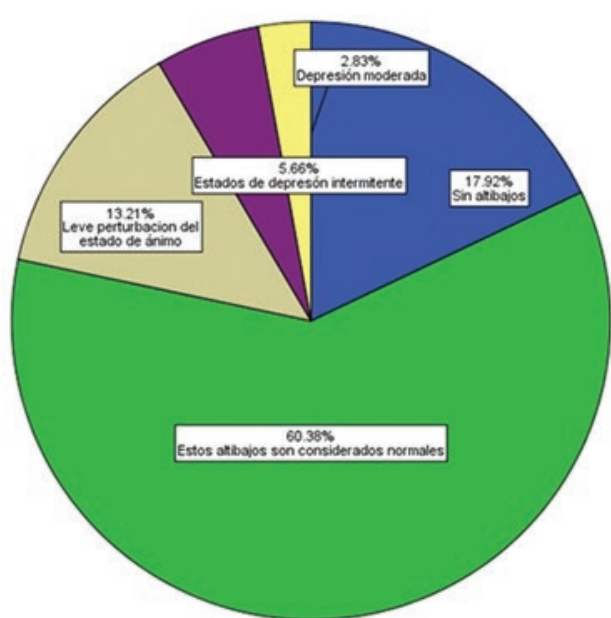

Figura 2. Distribución de los pacientes según el cuestionario de Beck.

una edad promedio menor (25.2 vs. 31.2 años) y un mayor número de cicatrices promedio (9.9 vs. 3.24). Estos hallazgos son concordantes con los resultados obtenidos por Bock, et al. ${ }^{10}$ y Balci, et al. ${ }^{11}$ en sus estudios. Adicionalmente, aunque se observó una mayor proporción de cicatrices en áreas visibles en el grupo de pacientes con deterioro importante de la calidad de vida (57.14\% vs. $48.3 \%$ ) en comparación con el grupo que no presentó afectación de la calidad de vida, estadísticamente no se demostró una diferencia significativa. Dicho hallazgo contrasta con lo reportado por Brown, et al. ${ }^{12}$ y por Lawrence, et al. ${ }^{13}$, quienes refieren haber encontrado mayor deterioro de la calidad de vida en pacientes con cicatrices en áreas visibles, como la cara. Una explicación que sustenta nuestros hallazgos es que las cicatrices en áreas cubiertas por la ropa son importantes para la intimidad sexual, al igual que las cicatrices en la cara lo son para la imagen que el individuo proyecta en su vida diaria.

El concepto de calidad de vida se basa en conocer la percepción del individuo de su posición en la vida y el contexto de su estilo de vida, vivienda, satisfacción en la escuela y en el empleo, así como la situación económica en la que se encuentra. Dicho concepto incluye la salud en la esfera biológica, psicológica y social. Por ello, la percepción de la calidad de vida dependerá del sistema de valores, estándares o perspectivas de cada individuo ${ }^{14-16}$. En este contexto, la mayoría de los pacientes con cicatrices buscan tratamiento tanto para disminuir los síntomas de dolor, prurito y restricción de la movilidad como para revertir la alteración cosmética que las cicatrices les genera ${ }^{17}$. Es un hecho que el deterioro de la calidad de vida atribuible a las cicatrices queloides es comparable al que presentan pacientes con otras enfermedades dermatológicas, como la psoriasis ${ }^{11}$. Brown, et al. ${ }^{12}$ identificaron cinco áreas de la calidad de vida afectadas en pacientes con diferentes tipos de cicatrices: confort físico y funcionamiento, aceptabilidad para sí mismo y para los demás, funcionamiento social, confianza en la naturaleza, manejo de la enfermedad y estabilidad emocional. En su estudio, la mayoría de los pacientes no estaban conformes con la apariencia de las cicatrices debido a su estigma y las asociaciones psicológicas percibidas. Sin embargo, nuestro estudio demuestra que el deterioro de la calidad de vida atribuible a las cicatrices queloides fue mínimo en nuestra muestra de pacientes, la cual es representativa de los adultos que acuden al dermatólogo. Es importante señalar que nosotros no incluimos pacientes con cicatrices hipertróficas ni por quemaduras, las cuales pueden ser más extensas y ocasionar una alteración cosmética mayor cuando se desarrollan en zonas como la cara y el cuello.

Finalmente, cuando analizamos los resultados de la aplicación del cuestionario de Beck para detección de rasgos depresivos encontramos que los pacientes que reportaban síntomas compatibles con depresión moderada presentaban en promedio un deterioro de la calidad de vida calificado como muy grande. Estos resultados podrían interpretarse de dos formas: la primera es que el constructo de calidad de vida (al reflejar indiscutiblemente el grado de bienestar psicológico) siempre se correlacionará con la prevalencia de enfermedades psiquiátricas, mientras que la segunda es que realmente haya una asociación entre la presencia de cicatrices queloides y el deterioro del estado de bienestar psicológico del individuo que las padece. Por ello, se requieren estudios que investiguen si este tipo de cicatrices ocasionan de forma secundaria una alteración psicológica, o si el deterioro de la calidad de vida es atribuible únicamente a la patología psiquiátrica que presente el individuo.

Dentro de las limitaciones del estudio, y que deberían explorarse en otro tipo de estudios con otro diseño metodológico, se encuentra el no haber interrogado sobre otros factores desencadenantes de los síntomas de depresión que manifestaron los pacientes y que potencialmente serían factores de confusión para proponer una asociación entre el desarrollo de cicatrices queloides y la depresión.

Nuestro estudio demuestra que las cicatrices queloides tienen un efecto negativo significativo en la calidad de vida de los adultos jóvenes, siendo este similar a lo reportado en otras enfermedades dermatológicas. 


\section{Financiación}

Ninguna.

\section{Conflicto de intereses}

Ninguno.

\section{Bibliografía}

1. Davidson S, Aziz N, Rashid RM, Khachemoune A. A primary care perspective on keloids. Medscape J Med. 2009;11:18.

2. Clark JA, Turner ML, Howard L, Stanescu H, Kleta R, Kopp JB. Description of familial keloids in five pedigrees: evidence for autosomal dominan inheritance and phenotypic heterogeneity. BMC Dermatol. 2009:9:8

3. Verhaegen PD, van Zuijlen PP, Pennings NM, van Marle J, Niessen FB van der Horst CM, et al. Differences in collagen architecture between keloid, hypertrophic scar, normotrophic scar, and normal skin: an objective histopathological analysis. Wound Repair Regen. 2009;17: 649-56.

4. Basra MK, Fenech R, Gatt RM, Salek MS, Finlay AY. The Dermatology Life Quality Index 1994-2007: a comprehensive review of validation data and clinical results. Br J Dermatol. 2008;159:997-1035.

5. Biljard E. Kouwenberg CA, Timman R, Hovius SE, Busschbach J J, Mureau MA. Burden of keloid disease: a cross-sectional health-related quality of life assessment. Acta Derm Venereol. 2017;97:225-9.
6. Poetschke J, Reinholz M, Schwaiger H, Epple A, Gauglitz GG. DLQI and POSAS scores in keloid patients. Facial Plast Surg. 2016;32:289-95.

7. Wang YP, Gorenstein C. Assessment of depression in medical patients: a systematic review of the utility of the Beck Depression Inventory-II. Clinics (Sao Paulo). 2013:68:1274-87.

8. Reinholz M, Poetschke J, Schwaiger H, Epple A, Ruzicka T, Gauglitz GG. The Dermatology Life Quality Index as a means to assess life quality in patients with different scar types. J Eur Acad Dermatol Venereol. 2015;29:2112-9.

9. Chuan SY, Goh CL. The impact of post-acne scars on the quality of life among young adults in Singapore. J Cutan Aesthet Surg. 2015;8:153-8.

10. Bock O, Schmid-Ott G, Malewski P. Mrowietz U. Quality of life of patients with keloid and hypertrophic scarring. Arch Dermatol Res. 2006;297:433-8.

11. Balci DD, Inandi T, Dogramaci CA, Celik E. DLQI scores in patients whit keloids and hypertrophic scars: a prospective case control study. J Dtsch Dermatol Ges. 2009;7:688-92.

12. Brown BC, McKenna SP, Siddhi K, McGrouther DA, Bayat A. The hidden cost of skin scars: quality of life after skin scarring. J Plast Reconstr Aesthet Surg. 2008;61:1049-58.

13. Lawrence JW, Fauerbach JA, Heinberg L, Doctor M. Visible vs hidden scars and their relation to body esteem. J Burn Care Rehabil. 2004;25: 25-32.

14. Velarde-Jurado E, Ávila-Figueroa C. Evaluación de la calidad de vida. Salud Publica Mex. 2002;44:349-61.

15. Ardilla R. Calidad de vida: una definición integradora. Revista Latinoamericana de Psicología. 2003;35:161-4.

16. Finlay AY, Khan GK. Dermatology Life Quality Index (DLQI): a simple practical measure for routine clinical use. Clin Exp Dermatol. 1994;19: 210-6.

17. Robles DT, Berg D. Abnormal wound healing: keloids. Clin Dermatol. 2007;25:26-32. 